\title{
Magnetic fluidized bed for solid phase extraction in microfluidic systems
}

\author{
Iago Pereiro ${ }^{\text {abc } \dagger}$, Sanae Tabnaoui ${ }^{\mathrm{ab}^{\dagger}}$, Marc Fermigier ${ }^{\mathrm{d}}$, Olivia du Roure ${ }^{\mathrm{d}}$, Stéphanie Descroix ${ }^{\mathrm{abc}}$, Jean- \\ Louis Viovy ${ }^{\text {abc }}$, Laurent Malaquin ${ }^{\text {abc* }}$
}

${ }^{a}$ Laboratoire Physico Chimie Curie, Institut Curie, PSL Research University, CNRS UMR168, 75005, Paris, France

${ }^{\mathrm{b}}$ Sorbonne Universités, UPMC Univ Paris 06, 75005, Paris, France

${ }^{\mathrm{c} I n s t i t u t ~ P i e r r e-G i l l e s ~ d e ~ G e n n e s, ~ 75005, ~ P a r i s, ~ F r a n c e ~}$

${ }^{\mathrm{d}}$ Physique et Mécanique des Milieux Hétérogènes, ESPCI-ParisTech-CNRS, Paris, France

*Current address : CNRS, LAAS, 7 avenue du colonel Roche, F-31400 Toulouse, France

${ }^{\dagger}$ These authors contributed equally to this work

Corresponding authors: laurent.malaquin@laas.fr, jean-louis.viovy@curie.fr

\begin{abstract}
Fluidization, a process in which a granular solid phase behaves like a fluid under the influence of an imposed upward fluid flow, is routinely used in many chemical and biological engineering applications. It brings, to applications involving fluid-solid exchanges, advantages such as high surface to volume ratio, constant mixing, low flow resistance, continuous operation and high heat transfer. We present here the physics of a new miniaturized, microfluidic fluidized bed, in which gravity is replaced by a magnetic field created by an external permanent magnet, and the solid-phase is composed of magnetic microbeads with diameters ranging from 1 to $5 \mu \mathrm{m}$. These beads can be functionalized with different ligands, catalysts or enzymes, in order to use the fluidized bed as a continuous purification column or bioreactor. It allows flow-through operations at flow-rates ranging from $100 \mathrm{~nL} / \mathrm{min}$ up to $5 \mu \mathrm{L} / \mathrm{min}$ at low driving pressures $(<100 \mathrm{mBar})$ with intimate liquid/solid contact and a continuous recirculation of beads for enhanced target capture efficiencies. The physics of the system presents significant differences as compared to conventional fluidized beds, which are studied here. The effect of magnetic field profile, flow chamber shape and magnetic beads dipolar
\end{abstract}


interactions on flow regimes are investigated, and the different regimes of operation described. Qualitative rules to obtain optimal operation are deduced. Finally, an exemplary use as a platform for immunocapture is provided a limit of detection of $0.2 \mathrm{ng} / \mathrm{mL}$ for $200 \mu \mathrm{L}$ volume samples.

\section{Introduction}

Analyte capture on a solid surface has been integrated in microfluidic platforms, making possible the preconcentration and detection of a wide variety of inorganic, organic, biological molecules, and more complex living entities. With the objective of improving capture efficiency and capacity, several solutions have been proposed to maximize the surface to volume ratio and accelerate kinetics, including microchannel structuring ${ }^{1}$, porous polymer monoliths ${ }^{2}$ or beads packing ${ }^{3}{ }^{4}$. A further improvement can be obtained if the solid phase is mobile, enhancing mixing, exposing more available surface and optimizing stirring and geometrical parameters for different protocol steps. Accordingly, bead arrangement with membranes ${ }^{5}$, bead/analyte mixing with peristaltic micropumps ${ }^{6}$ or dynamically actuated magnetic plugs ${ }^{7}$ have been reported. These approaches, however, involve relatively low densities of capture particles. In the macro world, this limitation can be overcome by particle beds. They are extensively used in industry to induce interactions between a fluid and a dispersed solid, with applications ranging from chemistry and biochemistry to metallurgy ${ }^{8},{ }^{9}$. Their implementation in microfluidic chips, however, faces a number of difficulties.

Two main types of particle beds are distinguished depending on their mode of operation: packed and fluidized beds. Packed beds are formed by a cylindrical column filled with a suitable packing material, and permeated by gases, vapors or liquids. Such packed beds are used e.g. for heterogeneous catalysis, filtration and separation, ion exchange, immunocapture, affinity concentration or purification, chromatography, etc. ${ }^{10-11}$.

A fluidized bed is obtained when a fluid or a gas is pumped upward at a sufficient velocity through a bed of initially packed (but unbonded) solid particles. The drag force generated by the flow induces a lift force that counterbalances the weight of the particles, above a threshold of flow velocity. In this case the particles no longer stay in contact with each other, the particle bed expands and suddenly 
takes on fluid properties. This transition between packed and fluidized bed corresponds to the point of incipient fluidization (or fluidization point). At this point, considering the whole particle bed, the drag force exerted on the particles matches the bed pressure drop (gradient) induced by gravity. As the fluid flow increases, the fluidized bed expands vertically, increasing its porosity, while the pressure drop remains substantially constant ${ }^{12}$.

As compared to packed beds, fluidized beds offer clear advantages: (i) They allow higher mixing efficiency and temperature homogeneity, (ii) bed plugging and channeling are usually minimized due to the movement of the solids, and (iii) operating conditions require lower pressure drops and allow higher flow-rates. Due to these advantages, fluidized beds have been used extensively in industrial applications. Originally developed for catalytic cracking in the petroleum industry, they have nowadays become a versatile fluid-solid intermixing device ${ }^{13}$.

The unfavorable balance between pressure drop and flow-rate upon miniaturization has been a strong hindrance to the generalization of packed particle beds applications in microfluidics. Fluidized beds, which reduce constraints for these two criteria, should be more favorable than packed beds in microfluidic devices, and offer the potential of enhanced mass transfer and fast heterogeneous reactions at a micrometer scale. This could be particularly interesting in bioanalytical applications, in which concentration, purification, capture and elution from a complex matrix are critical, reagents are generally expensive, and samples can be of very low volume.

The literature on the microfluidic integration of fluidized beds, however, is scarce, and only a few attempts have been reported. In Manz et al., 2004 a gas flow is used to generate fluidization of a miniaturized dry particle bed. This article reports the first application, to our knowledge, of miniaturization through chip technology for dry powder handling ${ }^{14}$. The application was limited to the injection and transport of small amounts of dry, non-cohesive powder and was thus restricted to the use of a gas phase as a fluid carrier.

More recently, Alwahabi et al. ${ }^{15}$ reported the liquid fluidization of $30 \mu \mathrm{m}$ glass micro-particles in rectangular microfluidic channel (PDMS) with a cross-section of 400 um x 175 um. This work, however, failed to fluidize micro-particles with water and showed the importance of interfacial forces in the fluidization process. Despite its integration in a microfluidic device, this approach was thus 
limited to the use of large and dense particles in combination with ethanol as a fluidizing medium, to ensure the particle suspension stability and limit particle-surface interactions.

Doroodchi et al. have achieved fluidization of large $225 \mu \mathrm{m}$ glass particles in capillaries with an inner diameter above $0.8 \mathrm{~mm}^{16}$. This article provides intriguing insights about the hydrodynamic behavior of the bed, but it is still restricted to the use of large particles. Potic et al ${ }^{17}$ also published a miniaturized device for biomass gasification in hot compressed water. The authors demonstrate the possibility for the fluidized bed to be operated in rather harsh operating conditions ( $p>200$ bars, $\mathrm{T}>500^{\circ} \mathrm{C}$, supercritical water), but the experiments also involve large sand particles $(>60 \mu \mathrm{m})$ in $1 \mathrm{~mm}$ large reactors.

The approaches described above typically consist in a scaling down of the concepts used in more conventional macroscopic fluidization devices, where the implementation and stability of the fluidized bed relies on the balance between gravity forces on one side and the combination of buoyancy and hydrodynamic forces induced by the liquid flow on the other side. The very principle of gravity-based fluidized bed, however, is poorly suitable to miniaturization, because the hydrodynamic drag force scales (in crude mean field view neglecting interparticles interactions) with the radius $R_{p}$ of particles, whereas the gravity forces scale with $\mathrm{R}_{\mathrm{p}}{ }^{3}$. As a numerical illustration, the gravity force experienced by a $2.8 \mu \mathrm{m}$ magnetic particle (e.g. Dynal) is $\mathrm{F}_{\mathrm{g}}=9.7 \cdot 10^{-15} \mathrm{~N}$ whereas the expected drag force obtained from the Stokes equation on an isolated particle is $F_{d}=4.24 .10^{-11} \mathrm{~N}$ for a relative velocity of $1 \mathrm{~mm} / \mathrm{s}$. The achievable flow-rates thus decrease dramatically with a reduction of size of the particles, which has so far limited microfluidic applications.

In order to overcome these limitations, we propose a new device concept, based on magnetic forces rather than gravitational forces. Indeed, the possibility of magnetic fluidization in the absence of gravity has been demonstrated in free-fall flights with the use of Helmotlz coils by a NASA study in $2004^{18}$, but this was still implemented in a macroscopic format, with low magnetic field gradients and in a configuration not suitable for microfluidics.

The use of magnetic particles in microfluidic chips has been often reported in the literature for bioanalytical applications. A typical application is the first bead-labeling of the target of interest and its concentration in an area of the chip where the magnetic field is particularly strong for subsequent 
detection and/or analysis ${ }^{6,19}$. In this case, the capture of analyte by beads is usually performed off-chip or in a volume-limited mixing chamber of the chip. To overcome this limitation other works have focused on the flow-through capture of an analyte on chip by customizing the physical arrangement of the beads. This has for example been obtained with self-assembled magnetic bead chains either retained by the geometry of the channel ${ }^{20}$ or positioned in a microfluidic chamber with the guidance of magnetic patterns ${ }^{21}$. Some of these systems, involving static plugs of particles, were successfully used for multiplexed analysis ${ }^{22}$. A system involving a dynamical actuation of the particle plug was also proposed to increase reaction kinetics ${ }^{7}$. These systems, however, can accommodate only a relatively small number of beads (e.g. of the order of $2 \times 10^{3}$ for ${ }^{20}, 10^{4}$ to $4 \times 10^{4}$ for $^{21,23}$, and $2 \times 10^{5}$ for ${ }^{7}$ ) and therefore present a low capture capacity. Except for $^{21}$, they could also accommodate only moderate flow flow-rates, between a few $\mathrm{nl} / \mathrm{min}$ and $1 \mu \mathrm{l} / \mathrm{min}$. This combination of low binding capacity and flow-rate make them unsuitable for analytes at very low concentrations. Static plugs of magnetic beads inside microfluidic channels retained by external permanent magnets have also been reported but despite a high particle density and capture capacity, non-homogenous percolation mechanisms inside these structures could not allow high capture efficiencies at reasonable flow-rates ${ }^{23}$.

Quantitative comparisons between bead capacities for different systems are difficult and should be taken with caution. First, different studies have used beads of different sizes, which change the available area per bead. Second, the operationally most useful capacity, which is the total number of functional ligands, will depend on a case by case on numerous biochemical factors such as ligands grafting density, orientation, affinity, avidity, and so on.

Here we show that beds of magnetic particles in the size range 1 to $5 \mu \mathrm{m}$, commercially available and routinely used for analyte separation protocols, can be fluidized in microdevices. These devices provide bead capacities- above $3.4 \times 10^{6}$ beads, which is one to several orders of magnitude higher than previously published systems. We believe that the gain in bead capacity, in our system, is high enough to allow anticipation of a much larger biochemical capacity in most applications.

Fluidization is obtained thanks to permanent magnets. In suitable configurations, forces of typically $\sim 10^{-11} \mathrm{~N}$, much higher than gravitational/buoyant forces, can be applied to micrometer-sized particles. These forces are sufficient to ensure particle confinement in a microfluidic device and to mimic the 
role of the gravity forces involved in macroscopic devices, while being able to counterbalance viscous drag up to operationally useful flow-rates. We investigated the influence of channel geometry, magnet position and pressure/flow-rate conditions in order to i) optimize the bed capacity and stability over a large range of flow-rates ii) ensure a uniform sample flow across the device and iii) favor the recirculation and mobility of the particles in the device to maximize sample/particle interaction. A first application of this system for bacteria culture and detection was recently reported ${ }^{24}$, and here we investigate the complex physics underlying its behavior. We investigated the operating conditions of the device and show its compatibility with a large range of applications involving sample preparation and analysis. As an illustration we show the applicability of this system for a biological protocol, achieving the extraction and elution of a model biomarker.

\section{Principle}

A major difference between gravity and magnetic forces, is that gravity directly derives from a field, and is thus operational in a constant field, whereas magnetic forces are dipolar in origin and depend on the field gradient. The positive consequence is that, for a given magnetization of the driving magnet (which is limited by technology); magnetic forces can be locally increased by increasing the divergence of the field, and thus the gradient, using a suitable magnet shape and position. A negative consequence of this required field non-uniformity, is that the force cannot be kept constant on arbitrary distances, so that obtaining a balance between confining and drag forces across the whole bed becomes an non-trivial challenge, in contrast with gravity-based beds, in which this is achieved in a simple channel with uniform section.

In previous work, our team proposed a device involving a straight rectangular channel flanked by two magnets $^{23}$. This geometry yielded a good confinement of the beads, but it created field inhomogeneities with maximum retainment forces on the channel's sides facing the magnets, and lower forces at the center, in which the flow velocity should be maximum following Poiseuille's law. This favored channeling within the bed along the channel's axis, with adverse effects on the kinetics of fluid-solid interactions. 
Since uniform magnetic forces cannot be achieved in extended space, a conceptual answer to the above problem is to co-optimize magnets and channels shape, to let both the local field strength (imposed by the magnetic element's geometry) and the local flow velocity (imposed by the channel's geometry) vary in a manner keeping to the best extent a constant ratio between drag and magnetic forces. As a second constraint, the dynamic equilibrium of the bed must be a stable one, i.e. upon a fluctuation bringing particles downstream, the balance between drag and retainment forces should vary in favor of the second. As we shall see, however, this second constraint is easily satisfied in the geometries explored here.

The design chosen to qualitatively fulfill the above specifications is described in Fig. 1 (a more detailed description of the device and setup can be found in the materials and methods section, and SI Fig. 1). First, the general direction of the magnetic field gradient should be collinear with the general flow direction, in order to maximize confinement efficiency. This is achieved by positioning an external permanent magnet in front of the flow entrance of the chamber. This yields a magnetic field gradient globally oriented upstream (from top to bottom in the figure, see SI Fig. 2 for a finite elements simulation of the magnetic streamlines inside the chamber), and decreasing towards the exit of the chamber (see section "influence of magnetic field distribution", below, for details). To compensate for this, and prevent particles to escape due to an excess of drag force, the channel is given a V-shape ensuring a decrease of flow velocity, and thus of drag force, from the entrance to the exit. Indeed, conical micro-fluid beds were already applied in the macro format, and are considered to suppress slugging and to favor particle mixing in regards to simple cylindrical beds. This geometry is interesting in particular when the particles tend to interact and agglomerate ${ }^{17}$.

The angle of the wall chamber relative to the central axis was set between 0 and $33^{\circ}$ and the height to $50 \mu \mathrm{m}$. (see section "Results and discussion", below, for details). A bent $100 \mu \mathrm{m}$ wide input channel avoids the return of beads towards the inlet when the flow is stopped: the combination of magnetic force and geometric confinement induces a compaction effect that turns the beads ensemble into a closely packed arrangement associated with low bead mobility. 


\section{Experimental}

\section{Chemicals and materials}

Devices were fabricated by pouring polydimethylsiloxane (PDMS, Sylgard 184, Dow Corning) on $\mathrm{PCB} /$ copper molds (Radiospares) containing positive-relief channels patterned in dry film resist (SY355, Microchem). PDMA-AGE (provided by Institute of Biocatalysis and Molecular Recognition, CNR, Milan, Italy) at $0.5 \%$ in water ${ }^{25}$ was used as a surface treatment prior to magnetic bed injection in the device. Superparamagnetic microparticles Dynabeads M-270 Carboxylic Acid and Dynabeads M-280 Sheep anti-Rabbit IgG (Thermo Fisher), with a diameter of 2,8 $\mu \mathrm{m}$ were used for hydrodynamic and immunocapture experiments, respectively. The buffer used was Phosphate buffered saline (PBS, Sigma Aldrich) containing $1 \%(\mathrm{~m} / \mathrm{v})$ of bovine serum albumin (lyophilized powder, Sigma Aldrich). Elutions were obtained with anhydrous citric acid (Sigma Aldrich) diluted to $0.1 \mathrm{M}$ in ultrapure water. For immunocapture, Rabbit anti-Mouse IgG Alexa Fluor ${ }^{\circledR} 488$ and CD1a Human IgG1, Alexa Fluor ${ }^{\circledR} 488$ (Thermo Fisher) were employed for positive and negative testing respectively.

\section{Fabrication of microfluidic device}

The masters for PDMS device fabrication were prepared according to the process described in our previous work ${ }^{26}$. Briefly, a $55 \mu \mathrm{m}$ thick photoresist dry film resist was laminated on a $50 \mathrm{~mm}$ copper PCB wafer. The chip patterns were then exposed through a mask (SELBA) to UV light by conventional photolithography and finally developed. The masters were directly used for the replication of structures by PDMS casting. The resulting $3 \mathrm{~mm}$ thick PDMS replica was then bonded to a $100 \mu \mathrm{m}$ thick PDMS layer that was previously spin coated on a $180 \mu \mathrm{m}$ thick glass slide. The bonding was performed by activation of both sample surfaces through oxygen-plasma treatment.

A surface treatment with PDMA-AGE $0.5 \%{ }^{25}$ was finally performed by incubating the polymer solution inside the chip for one hour followed by water rinsing and drying. 
The design of the chip, as illustrated in SI Fig. 1, consisted of three inlets composed of two $200 \mu \mathrm{m}$ wide channels joining a larger middle channel of $1000 \mu \mathrm{m}$ at $45^{\circ} \mathrm{C}$. This main channel then narrowed to connect the main chamber of the chip to a curved channel (100 $\mu \mathrm{m}$ wide) that served as the constraint for the retention of magnetic beads. The main chamber presented a linearly varying width from the starting $100 \mu \mathrm{m}$ at the entrance to a maximum of $1.8 \mathrm{~mm}$ and a total length of $9.5 \mathrm{~mm}$. This chamber finally led to the output of the chip by an $11.5 \mathrm{~mm}$ long and $100 \mu \mathrm{m}$ thick channel that served as an internal resistance of the chip.

\section{Microfluidic setup}

As shown in SI Fig. 1 the three inlets were connected to independent reservoirs, all of them controlled by a solenoid valve (Burkert 6604) used in a switch-on/switch-off configuration. The fluid flow coming from the starting reservoirs was pressure-regulated with the use of a pressure controller with three independent pressure exits (MFCS ${ }^{\mathrm{TM}}$, Fluigent) and further feedback-regulated with the flow-rate measurements taken at the exit of the chip by a flow-rate sensor (Flowell ${ }^{\mathrm{TM}}$, Fluigent). The material chosen for all tubings was PEEK. A $80 \mathrm{~mm}$ long and $50 \mu \mathrm{m}$ in internal diameter PEEK tube was placed at the end of the system to impose the desired global resistance.

\section{Analysis workflow}

Prior to the injection of magnetic beads in the chip the whole system was filled with buffer (PBS $+1 \%$ BSA) and $50 \mu \mathrm{g}$ of M-270 Carboxylic Acid magnetic beads, (equivalent number of $3.5 \times 10^{6}$ beads) were washed three times in batch with the same buffer. The injection of beads was then performed by disconnecting the PEEK tube of one of the entrances. A pipette cone was then inserted in the flexible PDMS chip entrance half-filled with buffer. The magnetic beads were loaded inside the cone by pipetting them in a volume of around $30 \mu \mathrm{L}$. A small magnet placed below the chip was used to bring the beads inside the chip and into the main chamber. Finally, a NdFeB12 permanent magnet (N50, Chen yang Technologies) was placed close to the inlet, aligned with the axis of the main chamber. Permanent magnets are advantageous as they offer high magnetic field intensity values (around 1.47 Tesla). The dimensions of the magnet $(30 \times 20 \times 20 \mathrm{~mm})$ were chosen to provide a magnetic field 
oriented mainly along the channel axis and to minimize the lateral component of the magnetic forces. A separation of $2 \mathrm{~mm}$ was left between the outermost limit of the curved entrance channel of the chip and the border of the magnet. Once both beads and magnet were in place a fluid flow could be set to pass through the system.

\section{Particle tracking}

A bed with $1 \%$ of the total number of magnetic particles Cy5-labelled was used for fluorescence imaging with a flow-rate of $1.5 \mu \mathrm{L} / \mathrm{min}$. Fluorescence images were taken with an interval of $100 \mathrm{~ms}$ and analyzed with the PIVlab tool for Matlab ${ }^{27}$.

\section{Biomarker capture/elution}

The desired concentration of Rabbit anti-Mouse IgG Alexa Fluor ${ }^{\circledR} 488$ was obtained in PBS through a serial dilution and first injected in the chip, before bead insertion, for fluorescence intensity measurement. This calibration measurement was taken in the output channel of the chip $(110 \mu \mathrm{m}$ in width) with the use of a Nikon Ti-E inverted microscope (40x objective). After the formation of the fluidized bed (with $50 \mu \mathrm{g}$ of Dynabeads M-280 Sheep anti-Rabbit IgG), the same solution was injected at a flow-rate of $1 \mu \mathrm{L} / \mathrm{min}$ for immunocapture (although any entrance could be used for this purpose, we will refer to this entrance as 1). The total injected volume was either 20 or $200 \mu \mathrm{L}$. At the end of the injection step, the valve was closed and PBS $+0.1 \%$ BSA was injected through a second entrance (entrance 2) at $1.5 \mu \mathrm{L} / \mathrm{min}$ until $20 \mu \mathrm{L}$. The entrance valve was then closed and the bed let to completely compact for $2 \mathrm{~min}$. The valve of the remaining entrance (entrance 3) was then open together with the valve of entrance 1 . An eluent solution (citric acid $0.5 \%, \mathrm{pH}=2$ ) was then injected from entrance 3 for $2 \mathrm{~min}$, without opening of the fluidized bed (as its resistance was higher than the open entrance 1). This step avoids the opening of the bed for the elution step with a diluted eluent front. After this time, entrance 1 was closed with the resulting opening of the bed. An eluent volume of $0.15 \mu \mathrm{L}$ was allowed to flow through before the pressure was set to zero. The bed then compacted, filled with eluent, and was let incubate for $10 \mathrm{~min}$. After this time the bed was again opened for $4 \mathrm{~min}$ at $200 \mathrm{~nL} / \mathrm{min}$ and the resulting elution peak measured in the output channel. The pressure was again 
set to zero for a second $10 \mathrm{~min}$ calibration and the opening/measuring step was repeated. Biological controls were performed with CD1a Human IgG1, Alexa Fluor ${ }^{\circledR} 488$.

For capture rate evaluation, after the first calibration step, the fluorescence intensity was measured in the output channel during the capture step. This fluorescence was considered to be due to the passing of non-capture analyte. The intensity value was then compared with the calibration signal to obtain the capture efficiency as $\left(\mathrm{I}_{0}-\mathrm{I}_{\mathrm{C}}\right) / \mathrm{I}_{0}$ (with $\mathrm{I}_{0}$ being the calibration intensity and $\mathrm{I}_{\mathrm{C}}$ the intensity during capture).

\section{Results and Discussion}

\section{Flow regimes}

Two working regimes were observed depending on the input pressure. At low pressures, the particle bed was maintained in a static packed regime (Fig. 1a). When the input pressure increased beyond a certain threshold pressure a fluidization process occurred: a sudden expansion of the fluidized bed was observed, associated with an abrupt increase of the flow velocity. In this fluidized regime, a constant recirculation of particles moving downstream in the chamber axis region and coming back following the walls of the chamber was observed (Fig. 1b). These first results show that the behavior of the magnetically stabilized fluidized bed is qualitatively quite similar to that of more conventional gravity-driven devices. These characteristics and the main parameters affecting this magneticallydriven fluidization were investigated more quantitatively in the following sections. 
a

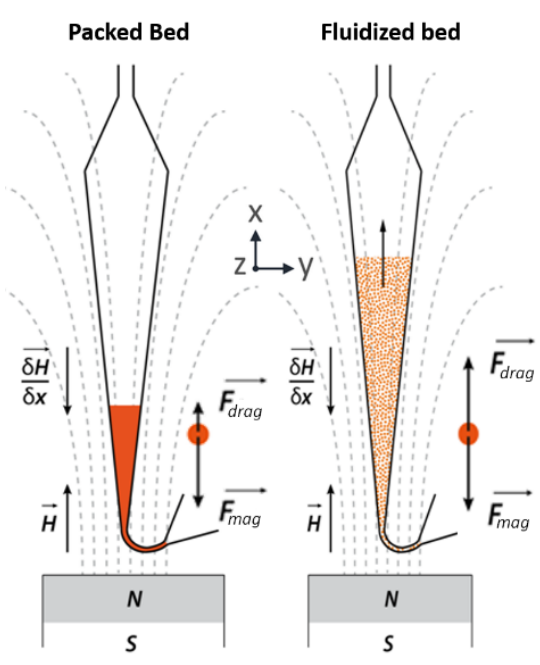

b

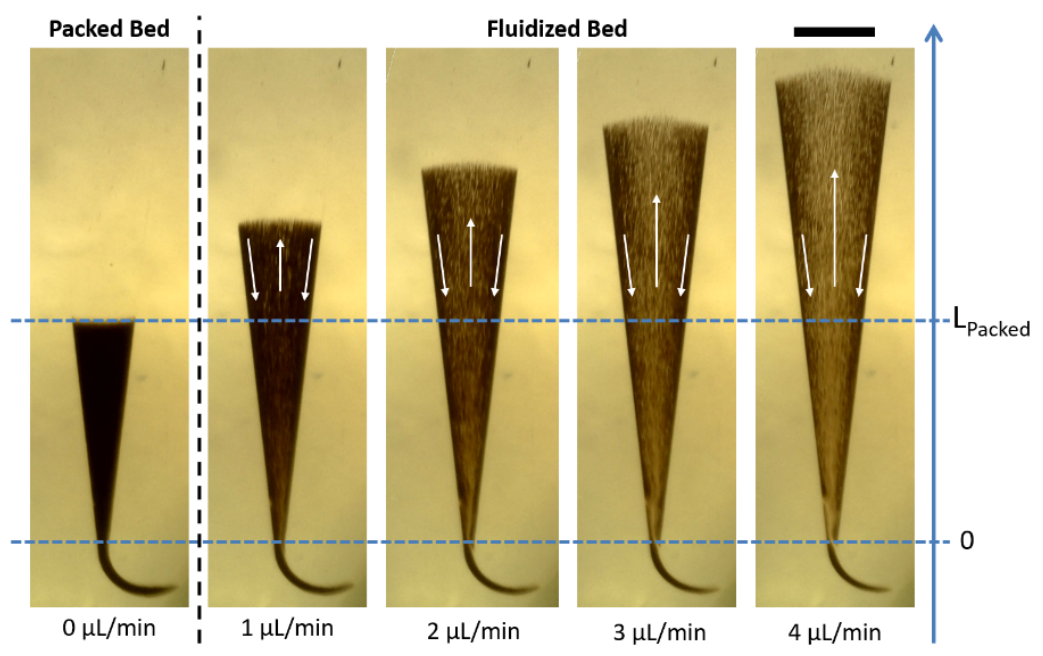

Figure 1. (a) Schematic illustrations of the microfluidic fluidized bed for two working regimes: at low imposed pressure particles are organized in a packed bed. Fluidization occurs when the pressure is sufficient to induce a flow fluid associated to drag forces that compensate for magnetic forces. Expansion of the bed volume occurs to maintain the equilibrium between drag and magnetic forces. (b) Images of the fluidized bed in the packed regime and fluidized for a series of flow-rates (scale bar $=1 \mathrm{~mm}$ ).

\section{Influence of chamber geometry}

We believe that both the homogeneity of the magnetic field orientation and the v-shape of the channel are essential to enhance the fluidization regime and favor particle recirculation through a spouting-like mechanism $^{12}$.

The vertical confinement imposed by the channel geometry $(50 \mu \mathrm{m}$ high) is also important as it favors the homogeneity of the fluid flow distribution across the channel. Whereas Poiseuille flow may arise in a square channel geometry, hydrodynamic simulations clearly show a rather uniform flow distribution across the v-shaped chamber along the y axis (SI Fig. 3). This means that the balance between magnetic and drag forces is homogeneous across the channel section and is mostly affected by the distance to the magnet. We believe that this feature is essential to maintain the homogeneity of the fluidization. 
The results on fluidization distribution of a series of chamber angles are shown in Fig 2a. Although fluidization was seen to be possible even with large angles, the expansion capacity of the bed was in this case severely limited, as the fluid tended to flow preferentially in the central region, resulting in a reduced bead mobility on either side. A chamber angle of $13^{\circ}$ was on the other hand seen to show a more homogeneous particle distribution and more efficient expansions, almost linear as a function of imposed flow-rate (Fig. 2b). This particular angle was initally chosen because of its similarity with the natural angle formed by preferential fluidization paths in confined channels (such as seen for $0^{\circ}$ in Fig. 2a). The rationale behind this choice was that, at first order, the bed in Fig $2 \mathrm{a}, 0^{\circ}$, could be seen as a self-organized fluidized bed with recirculation and quasi-uniform beads density in a cone with an angle of $13^{\circ}$, surrounded by a quasi-immobile bed, so that a fluidized bed with imposed solid walls situated at this $13^{\circ}$ angle would allow a recirculation similar to that of the center cone in the 0 angle device. This spontaneous angle indeed seemed to be optimal regarding its response to acceptable flowrates (in line with gravity-based beds), density uniformity and fraction of fluidized beads. Beds with a lower angle (not shown), also yielded good homogeneity, but sustained lower flow-rates before the onset of beads escape, so the angle of $13^{\circ}$ was the selected option for further studies. 


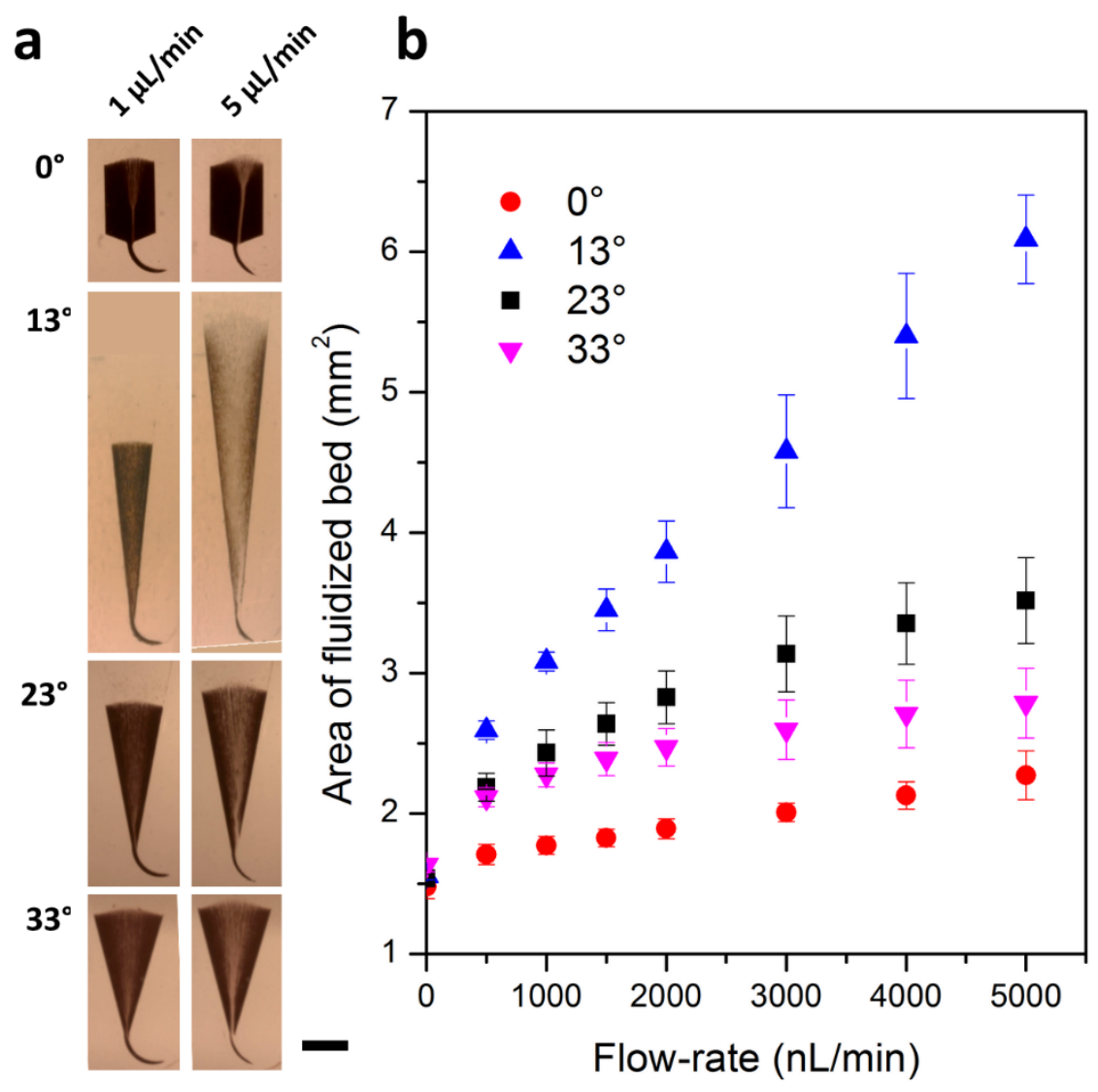

Figure 2. (a) Influence of chamber angle on fluidization behavior and (b) expansion as a function of imposed flow-rate $(n=3)$. A chamber angle of $13^{\circ}$ was seen to be optimal for the fluidization of a maximum of solid phase fraction (scale bar $=1 \mathrm{~mm})$.

\section{Influence of magnetic field distribution and beads dipolar interactions inside the chamber.}

Superparamagnetic beads are known to self-assemble in the form of chains and clusters in the presence of magnetic fields due to the magnetic pole interaction between the magnetic moments induced on the particles ${ }^{28}$. The resulting clusters tend to align parallel to the streamlines of the magnetic field. This behavior was reproduced in our system, where bead clusters formed and further aligned with the magnetic field independently of fluid flow (Fig. 3 and SI Fig. 4).

Fig. 3 shows the effect on bead distribution in the fluidized bed as a function of the position of the magnet in the $\mathrm{z}$ axis (perpendicular to the plane of the chamber). All the results seen in the previous section were obtained with a chamber z position $1 \mathrm{~mm}$ over the level of the bottom face of the magnet, a position considered the reference in the figure (Fig. 3a). The results show that a chamber closer to the axis of magnetization of the external permanent magnet led to more compact and hence less porous beds. This was partly due to the resulting increase in magnetic forces in the $\mathrm{x}$ direction, but also 
importantly due to the formation of longer clusters allowed by reduced inclination angles (the length of the cluster being limited in the $\mathrm{z}$ dimension by the height of the chamber, Fig. 3b). This led to an increase in particle cohesion to the point of impeding recirculation even at high flow-rates (Fig. 3a). In these conditions, a preferential channeling tended to appear in an otherwise compact bed (Fig. 3c). Assuming that the orientation of bead clusters was imposed by the direction of the magnetic field (as supported by high resolution images of the clusters, see SI Fig. 4) they translated in the chamber as rigid bodies. In the range of scales and velocities considered here, one may safely consider that flow boundary conditions are non-slip, so that they impose a unique flow velocity profile in their immediate vicinity at all depths in the chamber. This opposes the development of a Poiseuille profile, which would imply different fluid velocities in different streamlines. A minimum cluster angle is hence essential for fluidization.

Interestingly, though, increasing the $\mathrm{z}$ distance between the axes of the magnet and chamber resulted in more vertical magnetic streamlines, associated with shorter clusters (Fig. 3b). The magnetic recall force per cluster decreased, and this was accompanied by an increase in bed length and thus porosity (Fig. 3c). The reference position previously described (resulting in cluster angles of $\sim 35-45^{\circ}$ and thus $\sim 70-85 \mu \mathrm{m}$ in length) was found to be a good compromise between low void fraction and even porosity distribution. Other changes in magnet position only led to an increased void fraction (if magnet-chamber distance increased following the $\mathrm{x}$ axis), or uneven distribution and side compaction (y axis) 


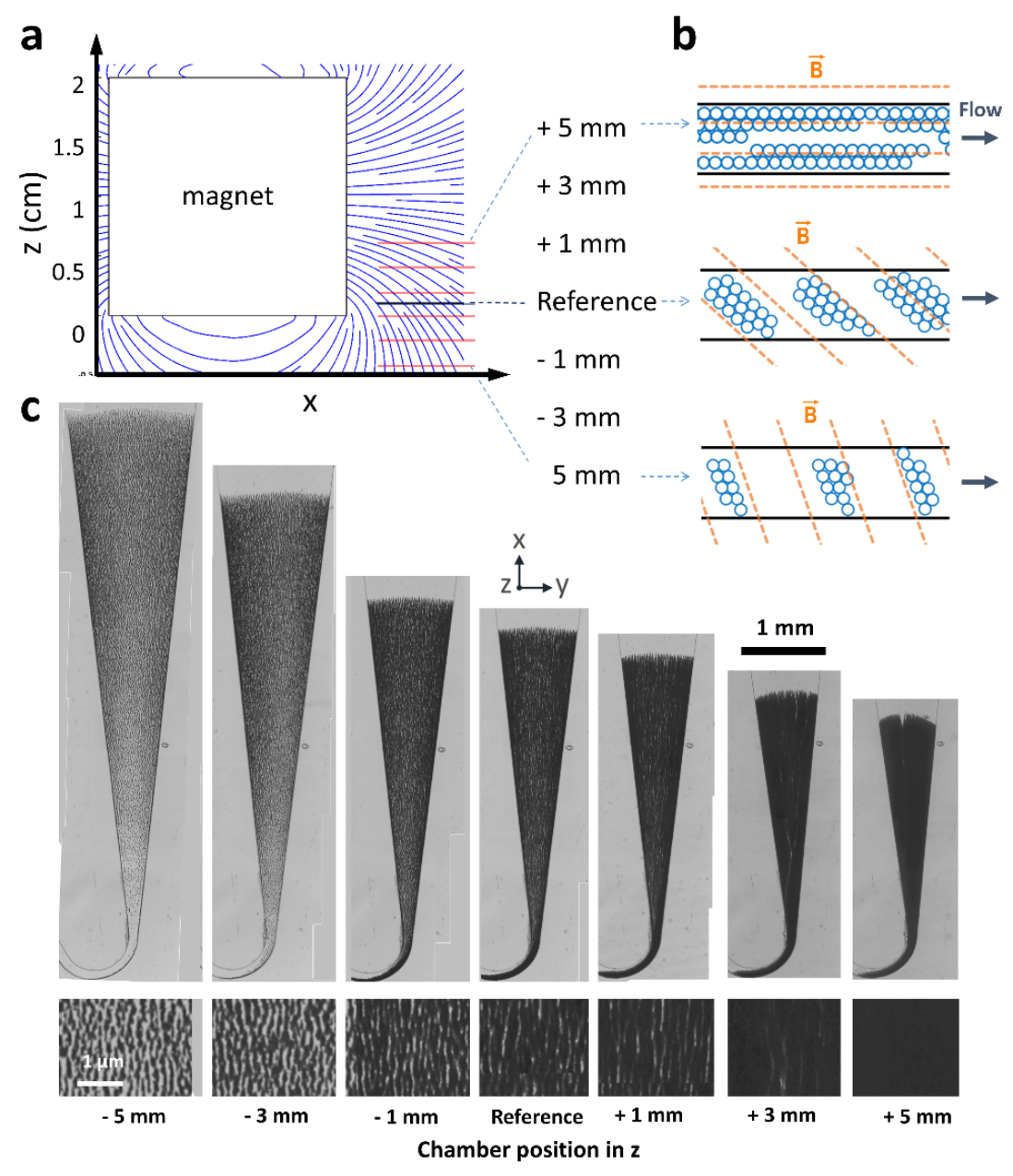

Figure 3. Effect of magnetic streamline angle and field gradients in the fluidization of the magnetic beads.

Higher angles lead to smaller clusters and higher porosity. Small angles result in channeling effects and no fluidization (1 $\mu \mathrm{L} / \mathrm{min}$ flow-rate for all images). Lower zoomed images taken equidistant from the entrance and the upper bed border.

\section{Hydrodynamic regimes}

With the resulting angle and magnetic field parameters, investigations were devoted to a study of the hydrodynamic behavior of the fluidized bed and of the operating conditions giving rise to a change in the bed regime. The inlet pressure in the device was imposed in the system and the corresponding flow-rate was measured downstream. The black curve in Fig. 4a shows the evolution of the flow-rate in an empty device i.e. where no particles were introduced. As expected, a linear dependence of the flow with pressure was observed, showing that the device behaved as a simple fluidic resistance. The other three curves were obtained after introducing three different quantities of microparticles in the system 25,50 and $75 \mu \mathrm{g}$ (corresponding to approx.. $1.66 \times 10^{6}, 3.33 \times 10^{6}, 5 \times 10^{6}$ particles respectively). 
The pressure vs. flow dependence of the corresponding bed was monitored in the 0-70 mbar range (see SI video 1 for the opening and closing process at $1 \mathrm{mBar} / \mathrm{s}$ for a $0-40 \mathrm{mBar}$ range).

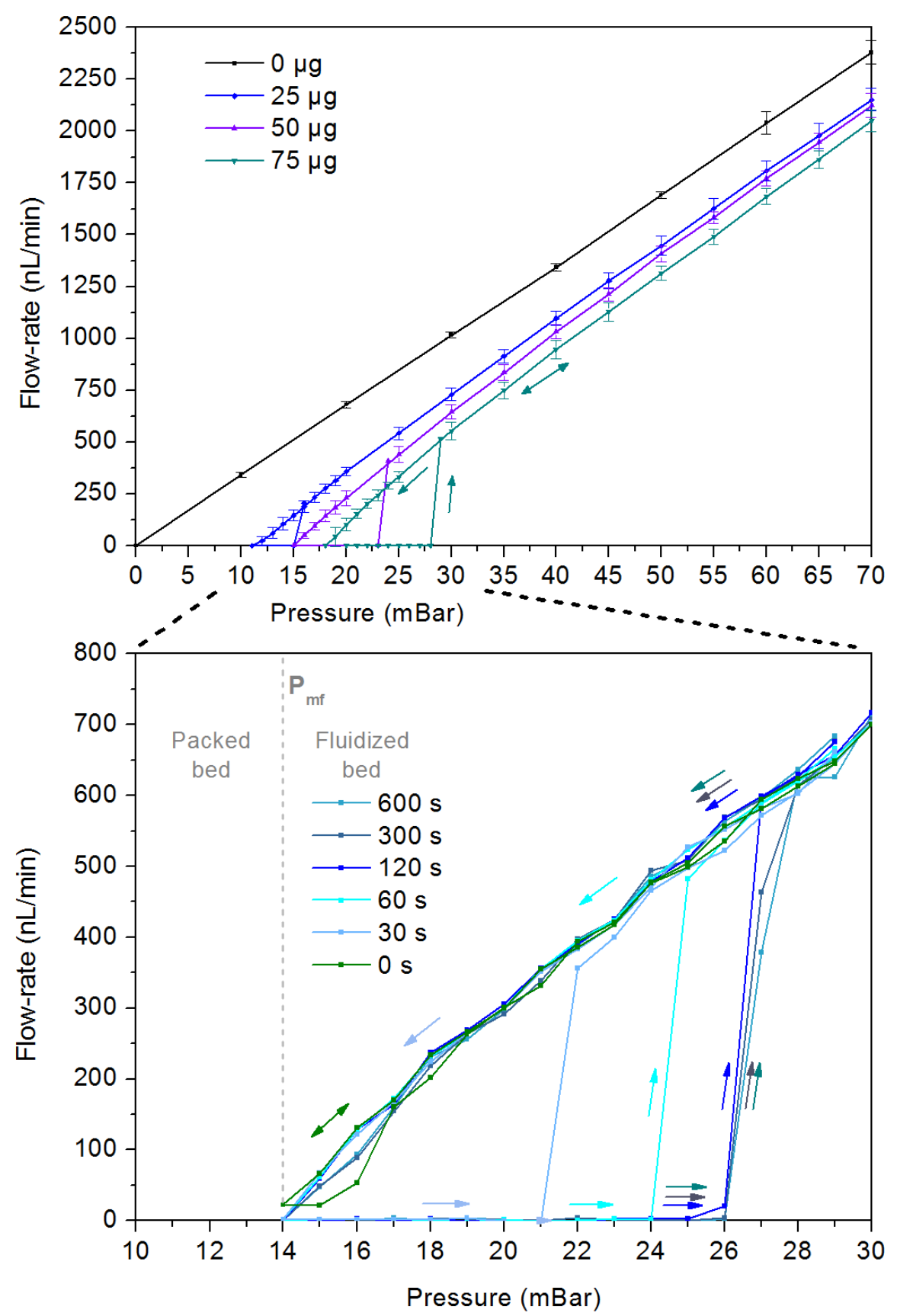

Figure 4. (a) Hydrodynamic pressure-flow-rate behavior of microfluidic magnetic fluidized bed for three different amounts of beads and for an empty chip (n=3). (b) Hysteresis observed for the effective fluidization pressure after different packing times for a single experiment.

Starting from a packed bed at 0 pressure, the pressure was increased at a constant rate of $1 \mathrm{mBar} / \mathrm{min}$. During this phase the flow-rate value remained below the sensitivity of the sensor $(<10 \mathrm{~nL} / \mathrm{min})$ meaning that the packed bed exhibited a very high hydrodynamic resistance. As shown by optical 
observations, the bed was in a packed state all along the pressure increase, indicating that in this regime, the drag force induced by the liquid flowing in the bed was not sufficient to compensate for magnetic forces and to destabilize the particle assembly.

When reaching a certain pressure $(15 \pm 2,24 \pm 2$ and $28 \pm 2 \mathrm{mBar}$ respectively for the masses of beads given before), the bed suddenly expanded causing an abrupt increase of the flow-rate. We will refer to this point as the pressure of effective fluidization $\left(\mathrm{P}_{\mathrm{ef}}\right)$. At this point, the hydrodynamic drag force overcomes the magnetic force plus interparticles interactions, destroying the compact state.

Not surprisingly, the pressure drop increased with the amount of particles introduced in the bed i.e. with the total magnetic force exerted on the bed (Fig 4a). As the pressure increased beyond the fluidization point, the bed expanded and the flow-rate in the fluidized bed follows a linear increase, with a slope comparable to the one obtained in the empty device (Fig. 4a). This intriguing result suggests that the particle bed induces in its fluidized regime a pressure drop quasi-independent of the flow-rate. This observation is consistent with the hypothesis that this pressure drop is directly related to the total magnetic force applied on the particles ensemble, which maintains the bed stability and compensates for viscous forces. In the case of gravity-based beds, this force can be considered as constant, giving rise to a constant pressure drop across the bed independently of flow-rate ${ }^{29}$. In our case, however, neither the magnetic field gradient nor the flow velocity were constant in the device. In particular, the magnetic force decreased in the $\mathrm{x}$ direction (SI Fig. 5), so that the total force applied on the bed was progressively reduced with the expansion of the bed, with an accompanying small reduction in its resistance. Recovering a behavior comparable to that of the gravity-driven bed, in this more complex situation, is thus intriguing, and deserves some comments. Taking into account the whole microfluidic system, the hydrodynamic resistance of the device can be described as the sum of i) the contributions of the empty microfluidic channels and ii) the pressure drop due to the viscous dissipation in the porous medium made by the fluidized particles (and equal to the total magnetic force exerted on the whole bed due to the conservation of momentum per unit cross-sectional area in a steady flow).

According to this, a very simple expression can be written for the pressure drop across the bed, if we further make the simplification that the porosity is constant across the whole volume of the bed: 


$$
\Delta P_{m f}=\frac{(1-\varepsilon)}{V_{p}} \int_{0}^{L} F_{m a g / p a r t} d x
$$

Where $\varepsilon$ represents the porosity of the bed (considered homogeneous), $\mathrm{V}_{\mathrm{p}}$ is the volume of a magnetic particle, and $\mathrm{F}_{\mathrm{mag} / \mathrm{part}}$ is the magnetic force applied on each particle (force considered constant for each transversal section of the bed but varying in $\mathrm{x}$ ).

We used this expression and numerical simulations to corroborate the experimental observations. The $\mathrm{x}$-component of the magnetic force on the particles was obtained from the magnetic field gradient in the chamber (finite elements simulations of the magnetic field distribution (COMSOL), SI Fig 5) and magnetization curves of Dynal particles ${ }^{30}$, through the expression $\vec{F}=(\vec{m} \cdot \nabla) \vec{B}^{31}$. By applying this force in Equation 1, as well as the information of bed length and porosity extracted from experimental data, $\Delta \mathrm{P}$-flow curves were obtained (SI Fig. 7). In this case, experimental and calculated values agree for low flow-rate values, when the homogeneity of the bed is maximal. For higher flow-rates the assumption of constant porosity for the entire section and length of the bed becomes less true, resulting in slight deviations. In any case, the overall good agreement between experiments and calculated results confirms the hypothesis that the bed properties are mainly driven by the balance between hydrodynamic and magnetic forces.

\section{Pressure of minimum fluidization}

Once in the fluidization regime a progressive decrease of the flow-rate invariably resulted in a transition of the bed to a packed bed regime at pressures significantly lower than $\mathrm{P}_{\text {ef. }}$ We will refer to this pressure as the pressure of minimum fluidization, $\mathrm{P}_{\mathrm{mf}}$ (for $50 \mu \mathrm{g}$ of beads $\mathrm{P}_{\mathrm{mf}}=14 \pm 1 \mathrm{mBar}$ ). At this point the pressure imposed by the fluid was no longer sufficient to compensate for the total magnetic force of the bead ensemble. This pressure was constant as a function of the bead loading of the bed and can be closely approximated with equation (1). A compaction of the bed then took place (see end of SI video 1) and a higher imposed pressure $\left(\mathrm{P}_{\mathrm{ef}}\right)$ was needed if the bed was to be open again, resulting in a hysteric behavior. 
In order to validate the applicability of the fluidized bed in microfluidic devices, this feature of our system was more thoroughly studied by performing repeated fluidization and compaction steps (Fig. 4b). Hysteresis is indeed a relevant characteristic of fluidized bed devices, in particular in the case of interacting particles: aggregation processes may cause variations in the incipient fluidization parameters and hinder bed efficiency ${ }^{17}$. Figure $4 \mathrm{~b}$ shows the evolution of the hydrodynamic characteristics of a fluidized bed during opening and closing cycles, performed for a $50 \mu \mathrm{g}$ bed by increasing the pressure from 0 up to $100 \mathrm{mBar}$ at a $1 \mathrm{mBar} / \mathrm{min}$ rate and decreasing it down to 0 at the same rate. Delay times varying from 0 to $600 \mathrm{~s}$ were added before each new cycle.

This experimental analysis suggests a clear dependence of the incipient fluidization parameters on the packing process. The longer the bed remains in the packed state, the higher the pressure that must be exerted in order to reach the incipient fluidization. In the case of the experiments shown in Fig $4 \mathrm{~b}$ for a $50 \mu \mathrm{g}$ bed, the opening values varied from 21 to $26 \mathrm{mBar}$ depending on the packed bed time (note that these are results from a single experiment: for $n=3$ a mean value of $P_{e f}=24 \pm 2 m B a r$ was observed for times longer than $2 \mathrm{~min}$, reflecting variability between experiments). A simple hypothesis explaining these results can be made, taking into account the kinetics of establishment of particle-particle interactions in the packed state. While the bed is maintained in the packed state, geometrical rearrangements of the particle assembly and the establishment of interparticle cohesive and magnetic forces contribute to increase the bed compaction and stability. When switching to the fluidized regime, the force applied to the bed has to compensate for the magnetic pulling forces and for these additional interparticle forces, thus giving rise to an increase of the effective fluidization pressure value. When integrating the fluidized bed in an analytical workflow, this behavior has to be taken into account in order to provide a robust and repeatable set of parameters to initiate fluidization. Once open, the behavior of the bed is, however, independent of its history.

\section{Bed porosity}

Fig. 5a shows measurements of the $2 \mathrm{D}$ area of the chamber filled by the fluidized particles at different flow-rates for three loading amounts of magnetic beads. The total volume of the bed was then used to calculate its total porosity $(\varepsilon)$, the total amount of particles and their individual volume being known 
(Fig. 5b). Taking into account the approximate number of beads per mass indicated by the provider and comparing their expected total volume as perfect spheres with the size of the resulting compact bed, the void fraction of the packed state was found to be around $35 \%$. When switching to the fluidized bed regime the mean porosity immediately increased to approximately $45 \%$ and further increased according to a non-linear curve up to $70 \%$ for $2 \mu \mathrm{L} / \mathrm{min}$. For the three bead loading masses studied, remarkably similar and almost overlapping $\varepsilon$-flow-rate curves were found. This suggests that the porosity is relatively independent of the bead loading, and of its total length. As previously seen (Fig.2 and 3) the local porosity is mainly determined by the magnetic field distribution and the channel geometry. Bead-bead magnetic interactions are short range, and hydrodynamic interactions are screened on distances larger than the chamber thickness. In the fluidized state, beads in a fixed $\mathrm{x}$ layer do not "see" beads downstream, and their spacing should thus be independent of the number of loaded beads. If this local porosity is fixed, the increase in the total area of the fluidized bed seen in Fig. 5a when the bead loading is increased is indeed expected.

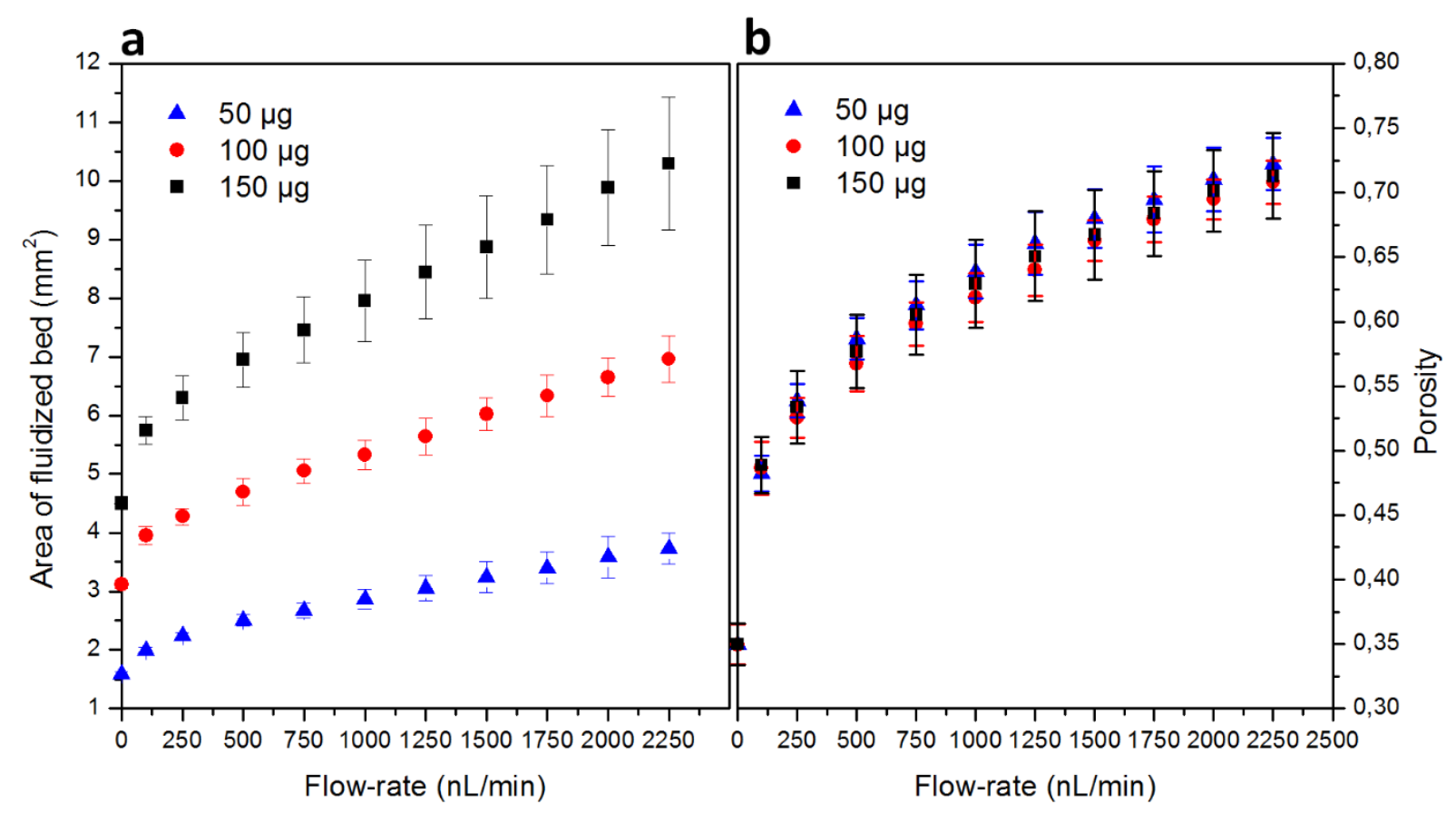

Figure 5. (a) Total area filled by the fluidized bed for three different amounts of beads as a function of flow-rate and (b) calculated void fraction of the fluidized bed as a function of flow-rate for the three different amounts of beads ( $n=3)$. 


\section{Particle recirculation inside the bed}

The previous calculations were made with the assumption that the porosity was homogeneously distributed within the bed. This is an approximation, as the constant recirculation of particles leads to a complex particle distribution that also depends on flow-rate. In order to characterize the motion of particles, image sequences of the particle bed were acquired in the fluidized regime and used for particle tracking followed by correlation analysis (Figure 6a). Both the flow distribution and particle motion in the fluidized bed were investigated for a fluidized bed containing $50 \mu \mathrm{g}$ of $2.8 \mu \mathrm{m}$ magnetic particles for a given flow-rate of $1.5 \mu \mathrm{L} / \mathrm{min}$.

a

b
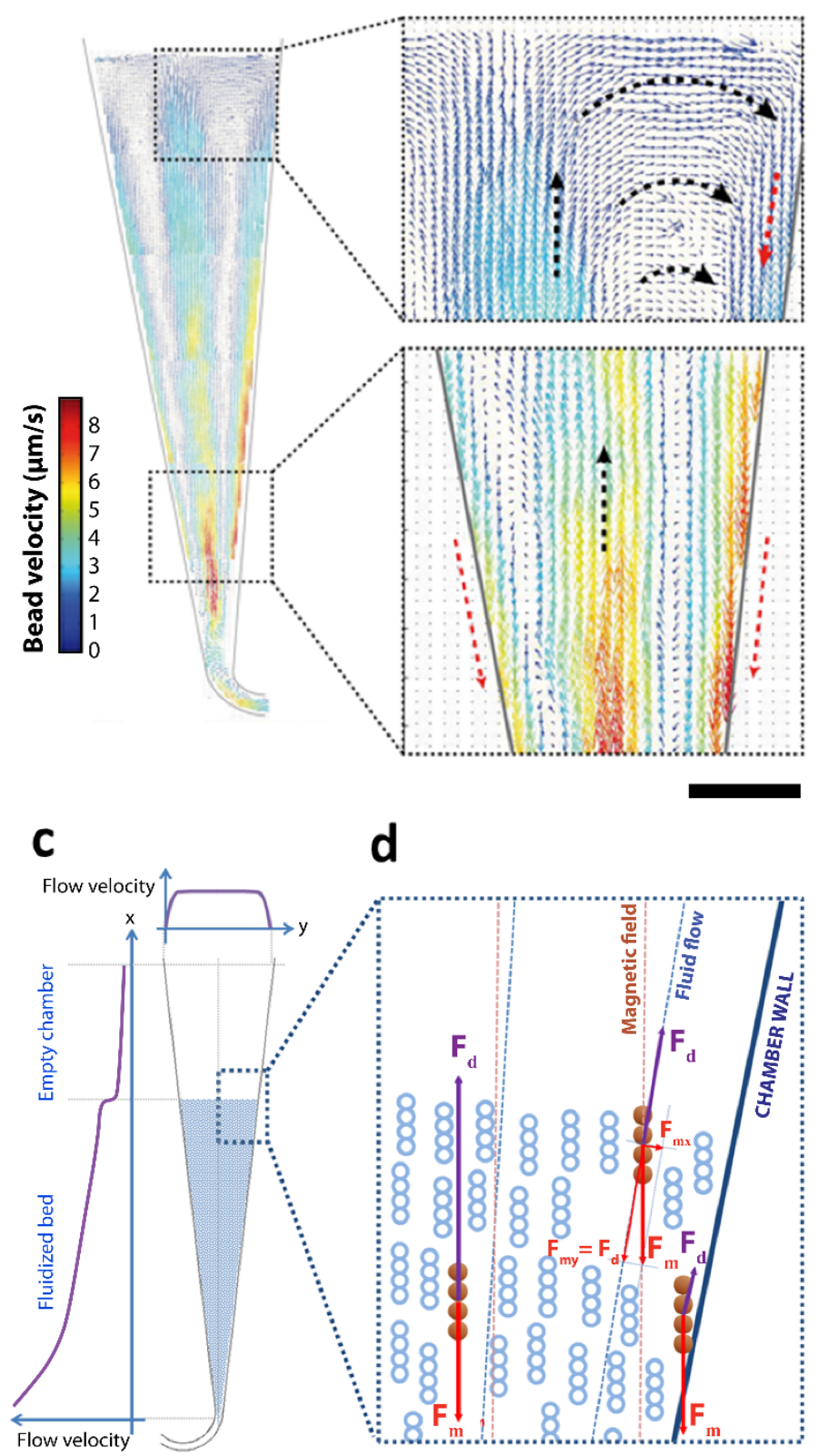
Figure 6. (a) Velocity field within the fluidized bed derived from particle tracking for a flow-rate of $1.5 \mu \mathrm{L} / \mathrm{min}$;

(b) detail of the sideward recirculation in the downstream limit of the bed, and of the opposite flow regions near the entrance; (c) schematic representation of flow velocity distribution in the longitudinal and transversal axis of the system and (d) indicative scheme of equilibrium of forces for the three main steps in bead recirculation

$$
(\text { scale bar }=100 \mu \mathrm{m}) \text {. }
$$

The results revealed a particle trajectory pattern comparable to the spouting regimes that may arise in macroscopic fluidized beds ${ }^{12}$. A flow of particles takes place in the center of the channel bringing the particles downstream. In this central region the particle velocity ranged from $8 \mu \mathrm{m} / \mathrm{s}$ close to the channel entrance down to 0 when reaching the terminal part of the fluidized bed. In this latter region, the particles were dragged away from the channel center towards the channel walls, according to an axisymmetric pattern (approximate velocity $1 \mu \mathrm{m} / \mathrm{s}$ ). A backward flow following the walls then brings the particles back towards the entrance of the chamber (approximate velocity $3-7 \mu \mathrm{m} / \mathrm{s}$ ). At the interface between the central and sidewall regions areas of low particle velocity were observed (Fig. $6 b)$.

This intriguing recirculation phenomenon can be explained, at least qualitatively, as illustrated in Fig. 6c and d. We hypothesize that in the central part, drag forces are dominant over magnetic forces. Particles are then dragged downstream along the channel main axis. When reaching the front of the fluidized bed a sudden drop in average flow velocity, due to the end of the solid fraction, abruptly reduces drag forces while the repelling magnetic force decreases more slowly, thus preventing particles to continue their downstream motion. The lateral displacement of particles can then be explained as the combined consequences of the particle inflow, which maintains the mass transfer balance, and the y-axis component of the magnetic field that increases linearly towards the sides of the chamber (Fig. 6d and SI Fig. 6). The return flow on the chamber's sides, finally, can be explained by the no slip boundary condition near the walls: in this region, the fluid velocity decreases significantly (SI Fig. 3) such that the upstream magnetic forces become dominant. As a result, a flow of particles is formed back towards the channel entrance. Simulations also suggest a sideward increase in angle difference between flow and magnetic streamlines, which results in an increasing lateral force close to the walls (Fig. 6d) and contributes to this recirculation mechanism. 
This mechanism ensures a mass transport balance and is thus essential to provide a stable and continuous recirculation of particles within the fluidized bed. It is particularly interesting in the context of biomarker capture and extraction as it provides a continuous and homogeneous recycling of particles and enhances their interaction with the fluid.

\section{Application to biomarker capture and pre-concentration}

Because of its flow-through nature, high surface to volume contact and high degree of mixing, this system should be ideally adapted for analyte preconcentration. Its potential for biomarker capture was tested by performing the immunocapture of a fluorescent model marker (Rabbit anti-Mouse IgG Alexa Fluor ${ }^{\circledR} 488$ ) with a $50 \mu \mathrm{g}$ bed of $2.8 \mu \mathrm{m}$ beads conjugated with specific antibodies (Dynabeads M-280 Sheep anti-Rabbit IgG). Fig. 7a shows the protocol followed. Briefly, a $20 \mu \mathrm{L}$ sample of biomarker in PBS was injected through the bed at a constant flow-rate $(1 \mu \mathrm{L} / \mathrm{min})$ for immunocapture. A washing step with buffer was then performed to remove non-specifically adsorbed species ( $1.5 \mu \mathrm{L} / \mathrm{min})$. After this step, a volume of eluent just sufficient to fill the porosity of the bed $(0.15 \mu \mathrm{L}$, citric acid $0.1 \mathrm{M})$ was injected, and an incubation of 10 minutes with the bed in the packed regime was performed. During this step a disruption of the antigen/antibody complex occurs due to the acidic $\mathrm{pH}$. After this time, a pressure above the fluidization threshold was applied to the eluent entrance. The biomarker was dragged downstream with the flow and its fluorescence measured by optical microscopy in the output channel.

Fig. $7 \mathrm{~b}$ shows a typical experimental result. The fluorescence of the biomarker before preconcentration was first measured in an empty channel (no beads, Fig. 7b1) and the elution peak obtained at the end of the protocol previously described was compared to this reference signal (Fig. 7b2). This allowed the estimation of preconcentration factors, which were found to be of $39 \pm 10$ for an initial capture volume of $20 \mu \mathrm{L}$ at $100 \mathrm{ng} / \mathrm{mL}$ and $485 \pm 150$ for $200 \mu \mathrm{L}$ at $10 \mathrm{ng} / \mathrm{mL}$ (Secondary elution peaks were obtained after reclosing of the fluidized bed for a second incubation step (Fig. 7b3). These values can be compared with performances reported on static plug by our group that were in the order of $80^{22}$. These secondary or further elution steps were used to estimate the efficiency of the first elution. The added 
peak intensity of these peaks was typically below $10 \%$ of the value of the first peak, indicating that the efficiency of the first elution was of the order of at least $90 \%$. To evaluate the immunoextraction efficiency, a $20 \mu \mathrm{L}$ highly concentrated solution $(20 \mu \mathrm{g} / \mathrm{mL})$ of non-specific target (CD1a Human IgG1, Alexa Fluor ${ }^{\circledR} 488$ ) was passed through the same bed composed of Dynabeads M-280 antiRabbit IgG and the protocol was repeated for preconcentration evaluation. In this case no significant eluent signal was detected beyond the LOD of the system demonstrating the specificity of the preconcentration approach. The capture efficiency was estimated to $85 \pm 3 \%$ (for $1 \mu \mathrm{L} / \mathrm{min}, \mathrm{n}=3$ ). This value was obtained from the comparison of fluorescence intensity measurements in the output channel with and without the presence of magnetic beads. A calibration curve was then obtained for the intensity of the elution peak in a range of concentrations of $1-100 \mathrm{ng} / \mathrm{mL}$ in $20 \mu \mathrm{L}$ samples. A linear correlation was obtained, showing the absence of saturation in this range of concentration, and the possibility to achieve good quantification values. A limit of detection of $0.2 \mathrm{ng} / \mathrm{mL}$ (three times the noise value) was found for these conditions.

These results show the potential of this system for preconcentration. It can be used with direct detection, as here, or as a front-end preconcentration step upstream of another analyte detection method. The inclusion of a washing step between capture and elution can also eliminate contaminants and reduce matrix effects, further contributing to lower LOD and improve reproducibility and specificity. Finally, in-situ detection can also be foreseen, for example by fluorescent marking or immunosandwich assays on the surface of the beads. 

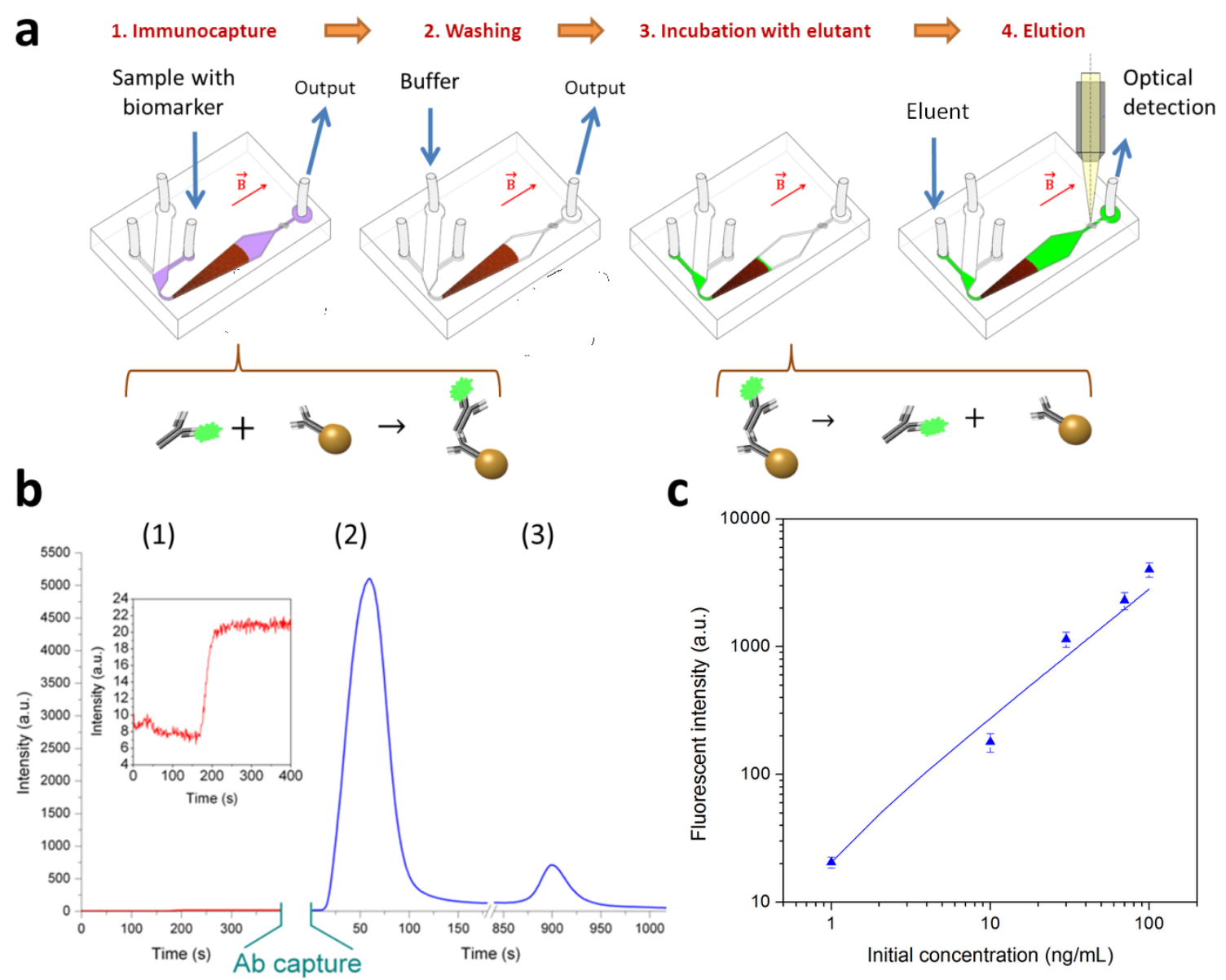

Figure 7. (a) Protocol followed for the concentration and extraction of a model biomarker by immunocapture followed by elution; (b) standard fluorescent measurements obtained with the immunocapture and elution of a $10 \mathrm{ng} / \mathrm{mL} 200 \mu \mathrm{L}$ sample, where 1) initial fluorescence of sample before preconcentration measured in the empty device, 2) first elution peak and 3) second elution peak are presented and (c) calibration curve obtained for a range of initial concentrations.

\section{Conclusion}

We investigated here the principle and the underlying physics of a new concept for microfluidic fluidized bed, using magnetic confining forces and commercial superparamagnetic particles in a size typically employed in biological applications (1-5 $\mu \mathrm{m}$ range) as the fluidized solid fraction.

On an operational ground, this system shares many features of standard gravitational fluidized beds, such as: i) the existence of packed bed and fluidized working regimes; ii) a pressure of minimum 
fluidization; iii) opening and closing hysteresis phenomena; iv) constant mixing of the solid phase, v) stable operation in a relatively large range of working flow-rates, and vi) combination of low interparticle distances ensuring efficient fluid-solid interactions, with a low pressure drop.

As compared to gravity-based systems, this new system involves a more complex physics, associated with the intrinsic non-uniformity of forces deriving from magnetic field gradients, the need to adapt accordingly the flow chamber to retain stable fluidized regimes, and the presence of magnetic dipolar interactions between the solid particles. Taking these specificities into account, however, it was possible to design an efficient and simple to construct device. It involves a straightforward chip design, easy to produce by soft lithography or more high throughput production methods, and a constant magnetic field obtained by an external permanent magnet. The different fluidization regimes, and the porosity and cluster size can be further adjusted on demand by commercially available pressure-based flow control systems.

As compared to previous microfluidic systems based on magnetic beads immunocapture, it allows a unique combination of beads capacity, flow flow-rate and low footprint, at comparable or better capture efficiency: $3.4 \times 10^{6}$ beads per chip, a flow throughput of $1.5 \mu \mathrm{L} / \mathrm{min}$ and capture efficiency better than $90 \%$, to be compared e.g. to 2000 beads, and a flow throughput around $1 \mathrm{nl} / \mathrm{min}$ for ${ }^{20}$ (quantitative capture efficiency not given); $1.3 \times 10^{4}$ particles and a flow throughput of $1.3 \mu 1 / \mathrm{min}$ for 23 (quantitative capture efficiency not given); and $2 \times 10^{5}$ beads, with a flow throughput of $60 \mathrm{nl} / \mathrm{min}$ and a capture efficiency estimated at $10 \%$ for $^{7}$.

Finally, an exemplary experiment involving immunocapture and elution demonstrates the feasibility of automated biological protocols with accurate liquid handling. Its stability, resistance to clogging and capacity of extraction of analyte from a liquid matrix, coupled to a very low volume of elution, make this system ideal for systems in which a preconcentration step is necessary for direct downstream analysis. 


\section{Acknowlegements}

This work was supported by a PhD grant from the Institut Pierre-Gilles de Gennes IPGG to IP, by ANR "Investissements d'Avenir" for Labex and Equipex IPGG, and by European FP7 programs (LOVEFOOD FP7-ICT-2011-317742, NAPES FP7-NMP-2013-604241, and ERC Advanced Grant CellO (FP7-IDEAS-ERC-321107),

\section{References}

1 M. A. Witek, S. D. Llopis, A. Wheatley, R. L. McCarley and S. A. Soper, Nucleic Acids Res., 2006, 34, e74.

2 C. Yu, M. H. Davey, F. Svec and J. M. J. Fréchet, Anal. Chem., 2001, 73, 5088-5096.

3 H. Andersson, W. Van Der Wijngaart, P. Enoksson and G. Stemme, Sensors Actuators, B Chem., 2000, 67, 203-208.

4 K. Sato, M. Tokeshi, T. Odake, H. Kimura, T. Ooi, M. Nakao and T. Kitamori, Anal. Chem., $2000,72,1144-1147$.

$5 \quad$ K. Y. Hwang, S. H. Kwon, S. O. Jung, K. Namkoong, W. J. Jung, J. H. Kim, K. Y. Suh and N. Huh, Anal. Chem., 2012, 84, 7912-7918.

6 K.-Y. Lien, J.-L. Lin, C.-Y. Liu, H.-Y. Lei and G.-B. Lee, Lab Chip, 2007, 7, 868-875.

7 Y. Moser, T. Lehnert and M. A. M. Gijs, Lab Chip, 2009, 9, 3261-3267.

8 D. Kunii and O. Levenspiel, ed. D. K. B. T.-F. E. (Second E. Levenspiel, ButterworthHeinemann, Boston, 1991, pp. 15-59.

9 V. Belgiorno, G. De Feo, C. Della Rocca and R. M. a Napoli, Waste Manag., 2003, 23, 1-15.

10 F. Vagliasindi and M. Benjamin, Water Sci. Technol., 1998, 38, 337-343.

11 K. Yao, M. T. Habibian and C. R. O’Melia, Environ. Sci. Technol., 1971, 5, 1105-1112.

12 R. G. Holdich, online B. http//www. Part. org. uk/particle_technology_book/particle_book. htm, Loughbrgh. Univ., 2003.

13 J. G. Yates, in Fundamentals of Fluidized Bed Chemical Processes, ed. J. G. Yates, Butterworth-Heinemann, 1983, pp. 121-159.

14 T. Vilkner, A. Shivji and A. Manz, Lab Chip, 2005, 5, 140-145.

15 V. Zivkovic, M. J. Biggs and Z. T. Alwahabi, AIChE J., 2013, 59, 361-364. 
16 E. Doroodchi, Z. Peng, M. Sathe, E. Abbasi-Shavazi and G. M. Evans, Powder Technol., 2012, 223, 131-136.

17 B. Potic, S. R. A. Kersten, M. Ye, M. A. Van Der Hoef, J. A. M. Kuipers and W. P. M. Van Swaaij, in Chemical Engineering Science, 2005, vol. 60, pp. 5982-5990.

18 T. Sornchamni, G. N. Jovanovic, B. P. Reed, J. E. Atwater, J. R. Akse and R. R. Wheeler, $A d v$. Space Res., 2004, 34, 1494-8.

19 B. Teste, F. Malloggi, J.-M. Siaugue, A. Varenne, F. Kanoufi, S. Descroix, Lab Chip, 2011, 11, 4207.

20 F. Lacharme, C. Vandevyver and M. A. M. Gijs, Anal. Chem., 2008, 80, 2905-2910.

21 A.-E. Saliba, L. Saias, E. Psychari, N. Minc, D. Simon, F.-C. Bidard, C. Mathiot, J.-Y. Pierga, V. Fraisier, J. Salamero, V. Saada, F. Farace, P. Vielh, L. Malaquin and J.-L. Viovy, Proc. Natl. Acad. Sci. U. S. A., 2010, 107, 14524-14529.

22 S. Bronzeau and N. Pamme, Anal. Chim. Acta, 2008, 609, 105-112.

23 A. Le Nel, N. Minc, C. Smadja, M. Slovakova, Z. Bilkova, J.-M. Peyrin, J.-L. Viovy and M. Taverna, Lab Chip, 2008, 8, 294-301.

24 I. Pereiro, A. Bendali, S. Tabnaoui, L. Alexandre, J. Srbova, Z. Bilkova, S. Deegan, L. Joshi, J.-L. Viovy, L. Malaquin, B. Dupuy and S. Descroix, Chem. Sci., 2016, 8, 1329-1336.

25 M. Chiari, M. Cretich, F. Damin, L. Ceriotti and R. Consonni, Electrophoresis, 2000, 21, 909916.

26 S. Miserere, G. Mottet, V. Taniga, S. Descroix, J.-L. Viovy and L. Malaquin, Lab Chip, 2012, 12, 1849-56.

27 W. Thielicke and E. J. Stamhuis, J. Open Res. Softw., 2014, 2, e30.

28 M. a Hayes, N. a Polson and A. a Garcia, Langmuir, 2001, 17, 2866-2871.

29 J. G. Yates, in Fundamentals of Fluidized Bed Chemical Processes, ed. J. G. Yates, Butterworth-Heinemann, 1983, pp. 199-211.

30 G. Fonnum, C. Johansson, A. Molteberg, S. Mørup and E. Aksnes, J. Magn. Magn. Mater., 2005, 293, 41-47.

31 Q. A. Pankhurst, J. Connolly, S. K. Jones and J. Dobson, J. Phys. D. Appl. Phys., 2003, 36, R167-R181. 